\title{
Making a better worm
}

\author{
Artyom Kopp
}

\section{The excretory systems of closely related worm species have distinct morphological and functional features. A new study now shows that evolutionary gain of lin- 48 expression in the excretory duct cell in Caenorhabditis elegans is responsible for the unique excretory system innovations present in this species.}

The genetic basis of phenotypic evolution is one of the most intriguing questions in biology $y^{1-3}$. Much of our recent progress in this field has used the candidate gene approach (Fig. 1). This approach leverages the power of model experimental systems by placing gene function in a historical context. Comparative analysis of gene expression, combined with the knowledge of gene function in a model organism, can be used to deduce the role of a gene in the origin of new phenotypes. But the final proof that the candidate gene does what we think it does cannot be obtained without a functional test: a genetic cross or, where crossing is impossible, transgenic analysis. On page 231, Wang and Chamberlin ${ }^{4}$ show how gene replacement can be used to distinguish between different models of phenotypic evolution.

C. elegans is distinguished from its nearest relatives by two unique features of its excretory system: a short excretory duct and high salt tolerance. Phylogenetic analysis showed that both features were derived and appeared only recently in C. elegans evolution (Fig. 1b) ${ }^{4}$. The development of derived traits in $C$. elegans depends on the expression of the zinc finger transcription factor $l i n-48$ in the excretory duct cell. In lin-48 mutants, both excretory duct morphology and salt tolerance revert to the ancestral condition (Fig. 1a) ${ }^{5}$. This prompted Wang and Chamberlin to examine lin-48 expression and function in other worm species. The results were notable. In the relatives of $C$. elegans that have long excretory ducts and low salt tolerance, lin-48 was not expressed in the excretory duct cell (Fig. 1b), and lin-48 transgenes from any of these species could restore the development of derived features in C. elegans lin-48 mutants (Fig. 1c). Together, these results suggested that evolutionary changes in the expression, but not the function, of lin- 48 had a key role in the origin of new morphological and physiological traits.

Artyom Kopp is in the Section of Evolution and Ecology and Center for Genetics and Development at the University of CaliforniaDavis, Davis, California 95616, USA. e-mail: akopp@ucdavis.edu

The exact nature of this role would not have become clear were it not for Wang and Chamberlin's asking the question: Would forcing the more primitive species to express lin-48 in excretory duct cells be sufficient to confer the derived traits? The answer was both yes and no. Expression of $l i n-48$ in the duct cell in C. briggsae, the closest relative of C. elegans, resulted in a C. elegans-like excre-

a

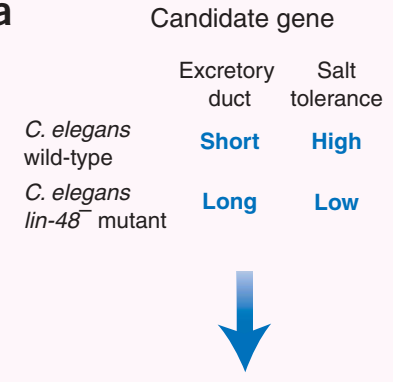

b Comparative analysis

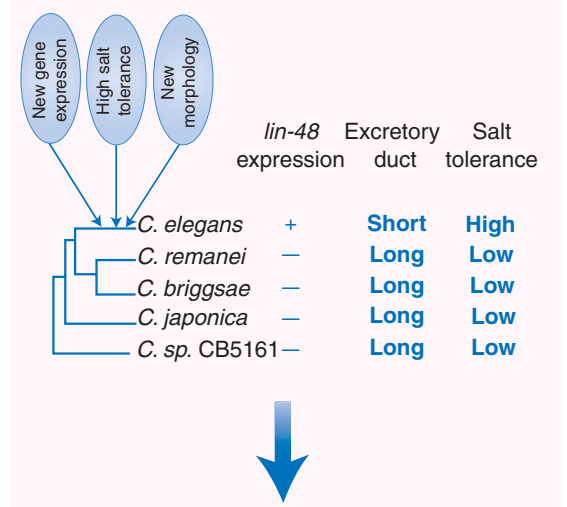

C

$\begin{array}{lccc}\text { C } & \begin{array}{l}\text { lin-48 } \\ \text { expression }\end{array} & \begin{array}{c}\text { Excretory } \\ \text { duct }\end{array} & \begin{array}{c}\text { Salt } \\ \text { tolerance }\end{array} \\ & + & \text { Short } & \text { High } \\ \begin{array}{l}\text { C. elegans } \\ \begin{array}{l}\text { C. briggsae } \\ \text { C. elegans } \\ \text { lin-48 mutant }\end{array}\end{array} & - & \text { Long } & \text { Low } \\ \begin{array}{l}\text { C. elegans lin-48- } \\ \text { rescued with }\end{array} & & \text { Long } & \text { Low } \\ \begin{array}{l}\text { C. briggsae lin-48 } \\ \text { C. briggsae } \\ \text { expressing lin-48 }\end{array} & + & \text { Short } & \text { High } \\ & & \text { Short } & \text { Low }\end{array}$

tory duct morphology but did not give C. briggsae a higher salt tolerance (Fig. 1c). A probable historical scenario is that a regulatory change in a single gene, lin-48, led to the evolution of new morphology, but other loci had to change before the worm could acquire greater salt tolerance.

\section{Single genes, big effects}

These results send both a promise and a note of caution to scientists who study the evolution of development. By the very nature of their discipline, developmental biologists are inclined to believe in, and look for, individual genes that account for a large proportion of phenotypic differences between species. Wang and Chamberlin's paper confirms once more that this is not a fool's errand: not only do such major-effect genes exist, but their existence can be proven conclusively by functional tests. At the same time, we are cautioned about the dangers of making historical inferences based solely on mutant phenotypes and gene expression patterns. Without the gene replacement tests, we would not have learned that the morphology and physiology of the worm excretory system are separable and follow different modes of evolution (monogenic versus polygenic).

Although the field of evolutionary developmental genetics is still too young to make sweeping generalizations, major-effect genes seem to be a common feature in morphological evolution. Sometimes, one such gene may completely account for the phenotypic differences between taxa. This is especially common for relatively simple traits, such as body

Figure 1 The 'candidate gene' approach to studying the evolution of development relies on a combination of genetic research in model organisms and comparative analysis. First, standard genetic techniques are used to identify the genes responsible for the development of the chosen trait in a model system, such as $C$. elegans (a). The expression of these genes is then analyzed in species with different traits to detect correlations between gene expression and phenotype (b). Hypotheses that emerge from comparative analysis can then be tested by functional assays, such as gene-replacement studies (c). 
pigmentation and cuticular decorations in insects and vertebrates ${ }^{6-9}$. More frequently, morphological differences involve several major-effect genes as well as weaker modifiers ${ }^{8,10-12}$. The existence of major-effect genes makes the evolution of animal morphology much more tractable at the molecular level than it would be under a highly polygenic model.

\section{Hopeful monsters}

Lest we start believing in hopeful monsters ${ }^{13}$, we should keep in mind the distinction between major-effect genes and major-effect mutations. A single locus may exert a substantial effect on the phenotype in interspecific genetic crosses or in transgenic animals. But there is no reason to think that this difference appeared all at once as a single mutation event. Rather, a gradual accumulation of many mutations, each with only a slight effect on the phenotype, may eventually turn a locus into what we perceive, in retrospect, to be a major-effect gene. When Wang and

\author{
William E Evans \& R Kip Guy
}

Chamberlin compared the cis-regulatory sequences of lin- 48 between C. elegans and its relative species ${ }^{4}$, they found extensive differences, including multiple nucleotide substitutions and deletions that affect putative binding sites for Ces-2, an important upstream regulator of $l i n-48$ (ref. 5). We do not know which of these molecular changes were responsible for the new expression pattern acquired by lin- 48 in the C. elegans lineage, but we are probably looking at a cumulative effect of multiple mutations.

The existence of major-effect genes tells us more about development than about evolution. There may simply be a limited number of developmental mechanisms that an animal can use to achieve a given phenotype ${ }^{9,14}$. Only a close synthesis of developmental biology with evolutionary and population genetics can help us understand how evolution explores these limited possibilities. Groups of closely related species of worms and flies have become a fertile ground for this synthesis ${ }^{15}$ and will no doubt continue to produce exciting results.
1. Carroll, S.B., Grenier, J.K. \& Weatherbee, S.D. From DNA to Diversity: Molecular Genetics and the Evolution of Animal Design (Blackwell Science, Oxford, Malden, Massachusetts, 2001).

2. Wilkins, A.S. The Evolution of Developmental Pathways (Sinauer Associates, Sunderland, Massachusetts, 2002)

3. Davidson, E.H. Genomic Regulatory Systems: Development and Evolution (Academic, San Diego, 2001).

4. Wang, X. \& Chamberlin, H.M. Nat. Genet. 36, 231-232 (2004)

5. Wang, X. \& Chamberlin, H.M. Genes Dev. 16 2345-2349 (2002).

6. Nachman, M.W., Hoekstra, H.E. \& D'Agostino, S.L. Proc. Natl. Acad. Sci. USA 100, 5268-5273 (2003).

7. Theron, E., Hawkins, K., Bermingham, E., Ricklefs, R.E. \& Mundy, N.I. Curr. Biol. 11, 550-557 (2001).

8. Wittkopp, P.J., Carroll, S.B. \& Kopp, A. Trends Genet. 19, 495-504 (2003).

9. Sucena, E., Delon, I., Jones, I., Payre, F. \& Stern, D.L. Nature 424, 935-938 (2003)

10. Swalla, B.J., Just, M.A., Pederson, E.L. \& Jeffery, W.R. Development 126, 1643-1653 (1999).

11. Naisbit, R.E., Jiggins, C.D. \& Mallet, J. Evol. Dev. 5, 269-280 (2003).

12. Takano-Shimizu, T. Genetics 156, 269-282 (2000).

13. Goldschmidt, R. The Material Basis of Evolution (Yale University Press, New Haven, 1940).

14. Gompel, N. \& Carroll, S.B. Nature 424, 931-935 (2003).

15. Simpson, P. Nat. Rev. Genet. 3, 907-917 (2002).

\title{
Gene expression as a drug discovery tool
}

\begin{abstract}
After establishing a pattern of gene expression that identifies a desired drug effect in leukemia cells (e.g., differentiation), post-treatment gene expression can be used to screen candidate compounds for their ability to induce the target phenotype.
\end{abstract}

Most medications currently used to treat cancer are relatively nonspecific cytotoxic agents, exerting effects on both tumor and normal tissue. The treatment of childhood acute lymphoblastic leukemia, for which cure rates have increased from $<10 \%$ in the 1960 s to over $80 \%$ today $^{1}$, can be attributed to the use of cytotoxic chemotherapeutic agents with low specificity. Unfortunately, such success is not evident in many human cancers, including acute myeloid leukemia (AML), where $<50 \%$ of affected individuals are cured with cytotoxic chemotherapy alone. New selective agents are needed for essentially all human

William E. Evans is Scientific Director of St. Jude Children's Research Hospital, Memphis, Tennessee, USA, and R. Kip Guy is Director of the Bay Area Screening Center of the California Institute for Quantitative Biomedical Research at University of California San

Francisco, San Francisco, California, USA. e-mail: william.evans@stjude.org

orrguy@cgl.ucsf.edu malignancies, to further improve cure rates and reduce host toxicity.

Advances in our understanding of cancer biology, human genetics, disease pathogenesis and molecular pharmacology hold great promise for the discovery of new approaches to treating cancer ${ }^{2}$. Progress and expectations have been heightened by initiatives such as the human genome project and by advances in technology that permit genome-wide analysis of cancer cells and facilitate the synthesis and high-throughput screening of large libraries of candidate compounds against molecularly defined targets ${ }^{3}$. The predominant strategy for identifying new anticancer agents is to screen for compounds that interact with specific molecular targets, rather than screening for predefined post-treatment phenotypes. Mechanisms that induce such phenotypic differentiation are normally complex and almost always involve the combined interaction of multiple signaling pathways. Although there has been considerable growth in the use of methods with high information content, ${ }^{4}$ the ability to detect a particular phenotype during a primary screen has lagged behind the ability to measure the functions of single molecular targets.

\section{A new twist}

On page 257, Kimberly Stegmaier and colleagues report a new strategy for screening compounds that force myeloid differentiation of AML cells ${ }^{5}$. Their approach is based on the observation that all-trans retinoic acid produces clinical remissions by inducing differentiation of acute promyelocytic leukemias harboring a mutated retinoic acid receptor alpha ${ }^{6}$. They developed a surrogate marker approach that uses post-treatment gene expression signatures in AML cell lines as the read-out for screening the ability of candidate compounds to induce differentiation of AML cells (Fig. 1). Microarray-based approaches have been previously applied to determining the mechanism of action of small molecules $^{7-9}$ or their toxicology ${ }^{10}$ and to assessing hormonal effects ${ }^{11}$. But the logistical reality of 